\title{
Nonstandard Echocardiographic Examination
}

\author{
Navin C Nanda, Aylin Sungur, Kunal Bhagatwala, Nidhi M Karia, Tuðba Kemaloðlu Öz \\ Division of Cardiovascular Disease, University of Alabama at Birmingham, Birmingham, Alabama.
}

(Cardiovasc. j. 2014; 6(2): 92-102)

\section{Introduction}

In addition to standard echocardiographic planes, there are many so-called nonstandard echocardiographic examination windows and planes that have been found useful in clinical practice. Standard planes have also been utilized with or without modification to find structures not routinely detected during echocardiographic examination. These are described below.

\section{Right Parasternal Examination Planes}

The patient is placed in the right lateral decubitus position in an attempt to slightly displace the lung to the right, and the transducer is positioned in the second to fourth right intercostal space very close to the sternum. Somewhat superior angulation is used to view the aortic root and ascending aorta, and this has been found useful in assessing aortic aneurysms as well as estimating the severity of aortic valve stenosis by color Doppler-directed continuous-wave Doppler interrogation or using a "standalone" Doppler probe. Inferior angulation facilitates examination of the superior vena cava as it enters the right atrium. The crista terminalis, a segment of the right coronary artery and the right atrial appendage, may also be viewed using this approach. This window is also useful to view the entire extent of the atrial septum, and both sinus venosus and secundum atrial septal defects are easily identified in this view by color Doppler because the flow signals are parallel to the ultrasonic beam. Because of this, accurate estimation of flow velocities through the defect can be obtained leading to more reliable estimation of shunt flow. Thrombi and other masses in the superior vena cava can be well evaluated. The inferior vena cava and the coronary sinus together with their valves and tributaries have also been visualized in this view. Pulmonary veins may also be observed entering the left atrium. In some patients, it has been possible to view the aortic arch and proximal descending aorta together with the surrounding structures utilizing this window. Examination using this approach is easier when the right heart or the ascending aorta is dilated as these results in further rightward displacement of the right lung producing a larger window. The two-dimensional study can be supplemented by live/real time, three-dimensional echocardiography, which may provide significant incremental information, and thus a more comprehensive assessment of various, normal anatomic structures and abnormal findings identified by this examination approach. Figures 1 to 14 show normal and abnormal findings obtained from the right parasternal approach using two-dimensional and live/real time, threedimensional echocardio-graphy. ${ }^{1-5}$

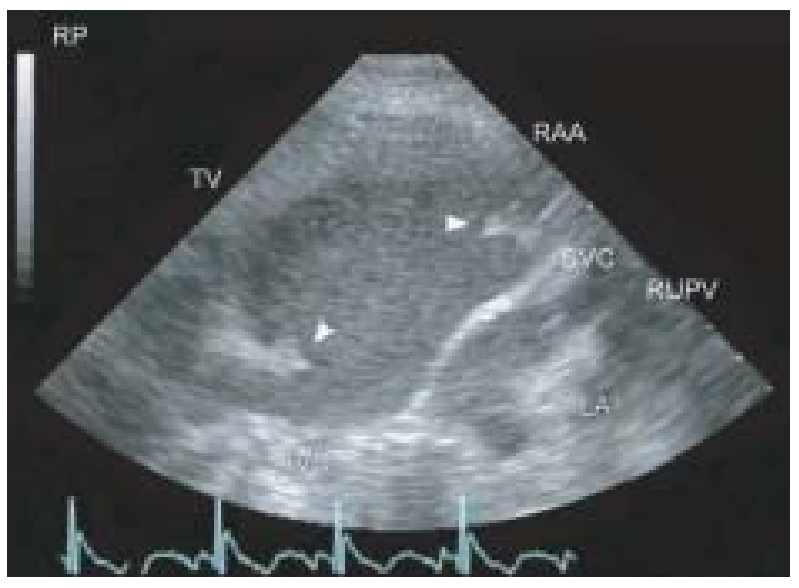

Fig.-1: Two-dimensional transthoracic echocardiography. Right parasternal approach (RP). The left arrowhead shows the Eustachian valve, and the right arrowhead points to crista terminalis. IVC, inferior vena cava; (LA: Left atrium; RAA: Right atrial appendage; RUPV: Right upper pulmonary vein; SVC: Superior vena cava; TV: Tricuspid valve).

(This article is reproduced with permision with reduced number of figures from the book 'Comprehensive Textbook of Echocardiography' edited by Prof. Navin C Nanda. )

Address of Correspondence: Prof. Navin C Nanda. Division of Cardiovascular Disease, University of Alabama at Birmingham, Birmingham, Alabama. 


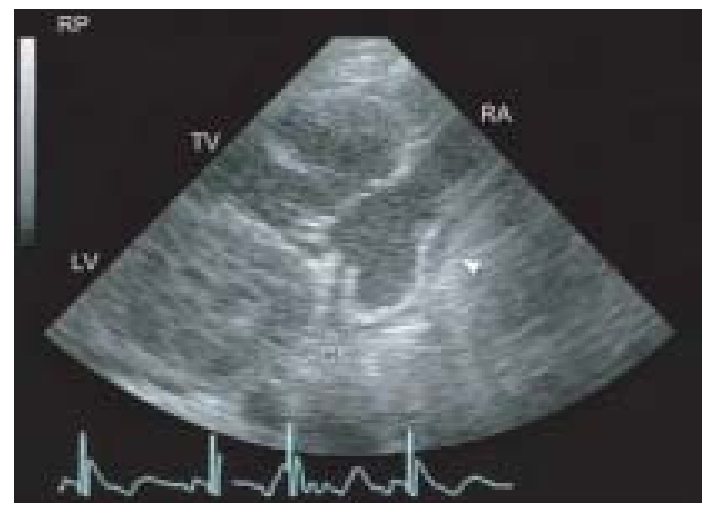

Fig.-2: Two-dimensional transthoracic echocardiography. Right parasternal approach (RP). The arrowhead shows the right marginal vein draining into the coronary sinus (CS); (LV: Left ventricle; RA: Right atrium; TV: Tricuspid valve).

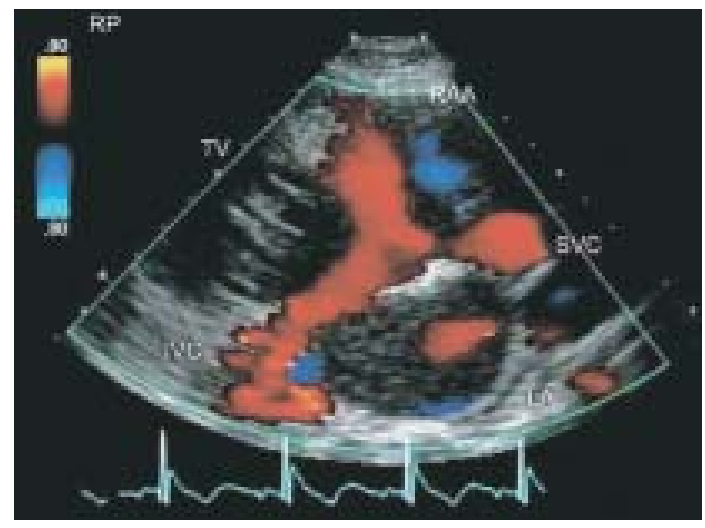

Fig.-3: Two-dimensional transthoracic echocardiography. Right parasternal approach (RP). Shows flow signals (red) moving from the superior vena cava (SVC) and inferior vena cava (IVC) into the right atrium. (LA: Left atrium; RAA: Right atrial appendage; TV: Tricuspid valve).

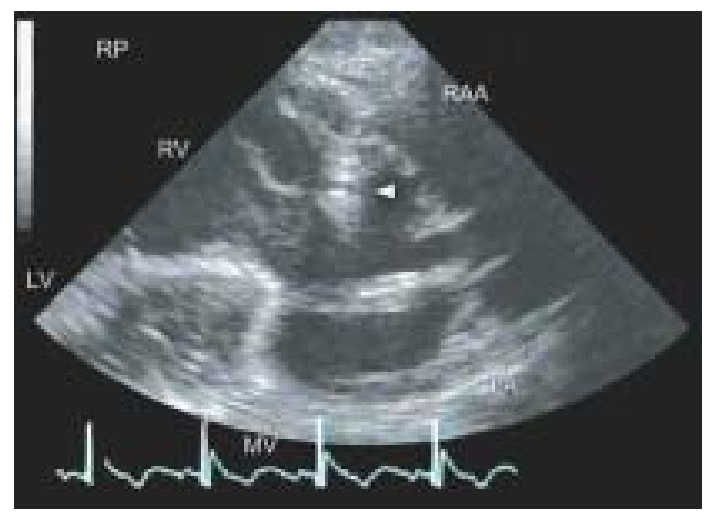

Fig.-4: Two-dimensional transthoracic echocardiography. Right parasternal approach (RP). The arrowhead points to right coronary artery in the right atrioventricular junction. (LA: Left atrium; LV: Left ventricle; MV: Mitral valve; RAA: Right atrial appendage; $R V$ : Right ventricle).

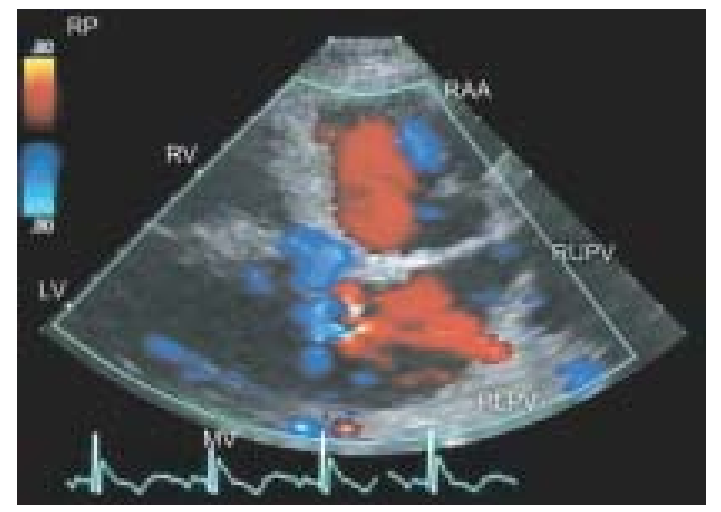

Fig.-5: Two-dimensional transthoracic echocardio-graphy. Right parasternal approach (RP) showing flow signals (red) moving from the right lower pulmonary vein (RLPV) to the left atrium. (LV: Left ventricle; MV: Mitral valve; RAA: Right atrial appendage; RUPV: Right upper pulmonary vein; $R V$ : Right ventricle).

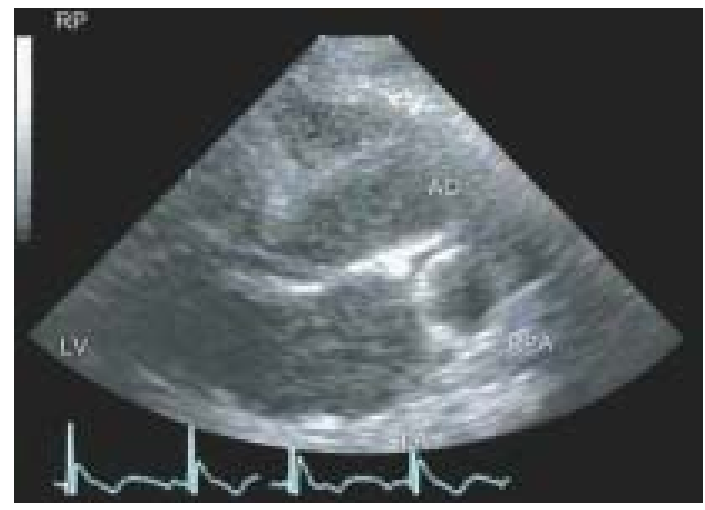

Fig.-6: Two-dimensional transthoracic echocardio-graphy. Right parasternal approach (RP). Shows an image of the ascending aorta (AO) using this window.

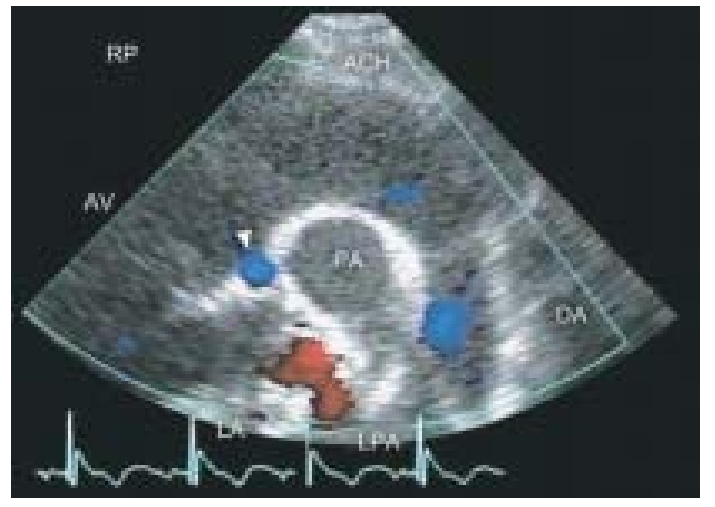

Fig.-7: Two-dimensional transthoracic echocardio-graphy. Right parasternal approach (RP). The arrowhead shows flow signals (blue) in the left main coronary artery between the aorta and the main pulmonary artery. 


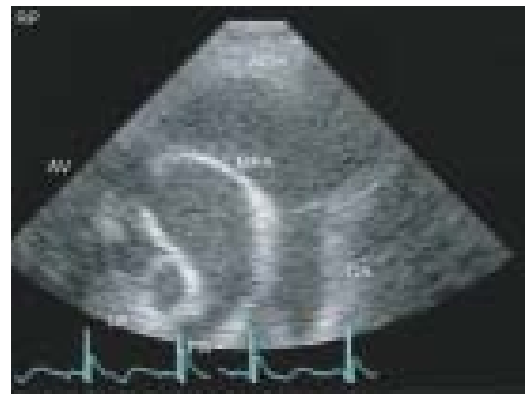

Fig.-8: Two-dimensional transthoracic echocardiography. Right parasternal approach (RP) showing the main pulmonary artery (MPA) and the left pulmonary artery (LPA) located below the aortic arch (ACH). (AV: Aortic valve; DA: Descending thoracic aorta; LA: Left atrium).

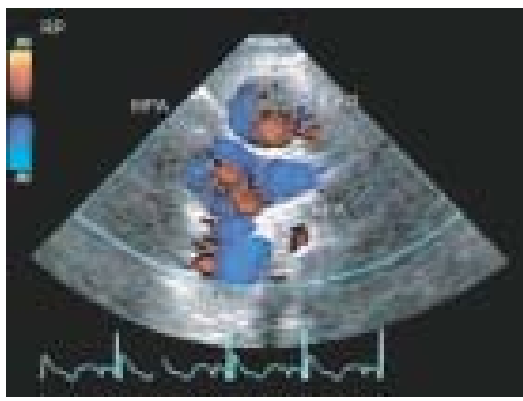

Fig.-9: Two-dimensional transthoracic echocardio-graphy. Right parasternal approach (RP) showing the main pulmonary artery (MPA) bifurcating into the left (LPA) and right (RPA) branches. (AO: Aorta; PV: Pulmonary valve).

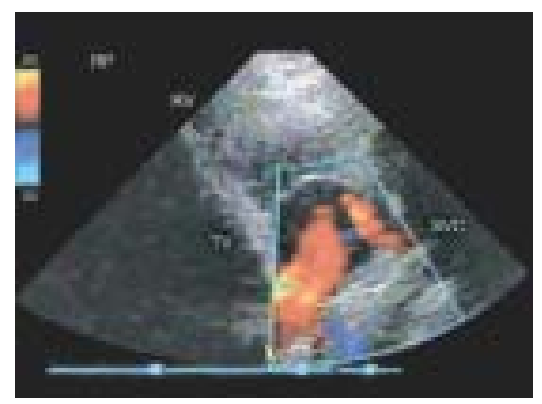

Fig.-10: Two-dimensional transthoracic echocardiography. Right parasternal approach (RP). Color Doppler examination shows normal flow signals moving from the inferior vena cava (IVC) and superior vena cava (SVC) into the right atrium. (RV: Right ventricle; TV: Tricuspid valve)

A
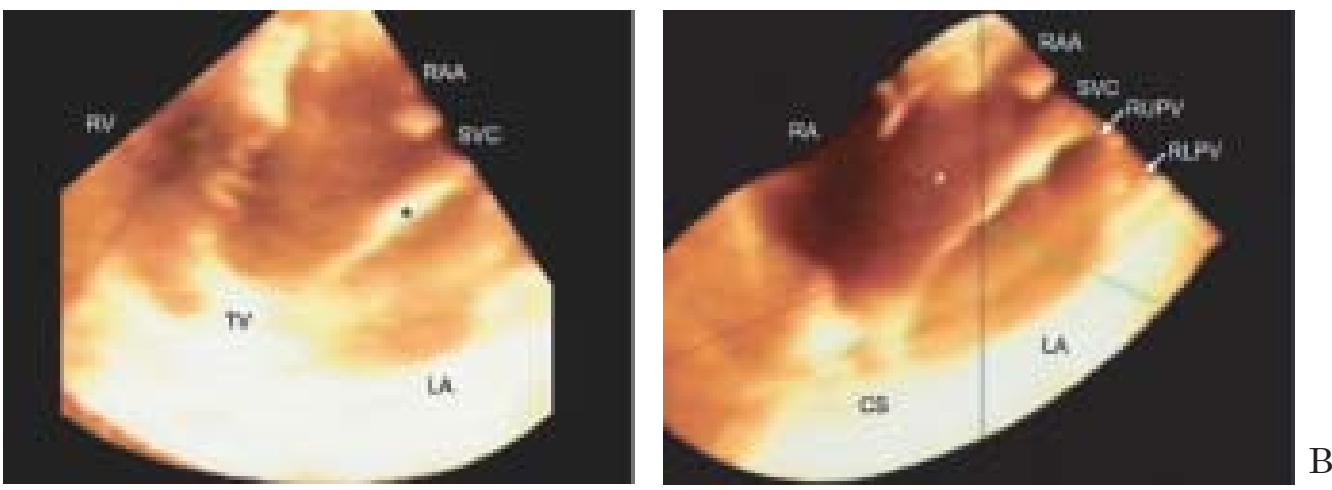

C
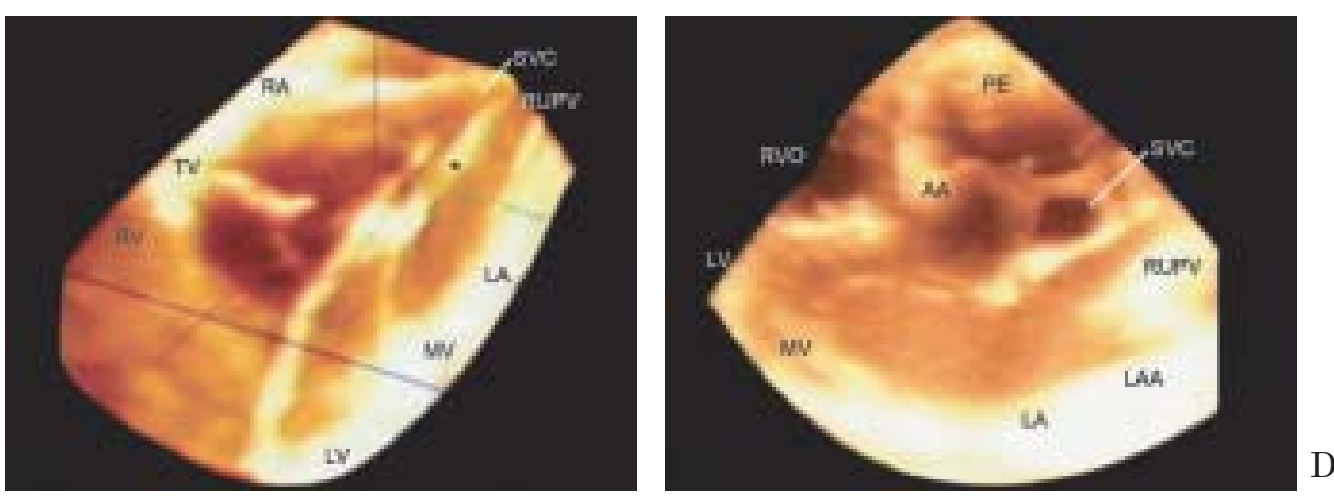

Figs.-11A to D: Three-dimensional right parasternal transthoracic echocardiographic examination of atrial septum and superior and inferior vena cava. (A) The atrial septum (*), the entrance of superior vena cava (SVC) into the right atrium, the base of the right atrial appendage (RAA), tricuspid valve (TV), and left atrium (LA) are shown; (B) The previous image has been tilted to view the atrial septum $\left(^{*}\right)$ en face and to more clearly demonstrate the entrance of both the right upper (RUPV) and the right lower (RLPV) pulmonary veins into the LA; (C) Mitral valve (MV), left ventricle (LV), and RUVP are brought into view by further cropping; (D) Another view demonstrating SVC in the short axis, long segment of RUPV, LA appendage (LAA), MV, LV, and ascending aorta (AA); Source: Reproduced with permission from reference 3. 

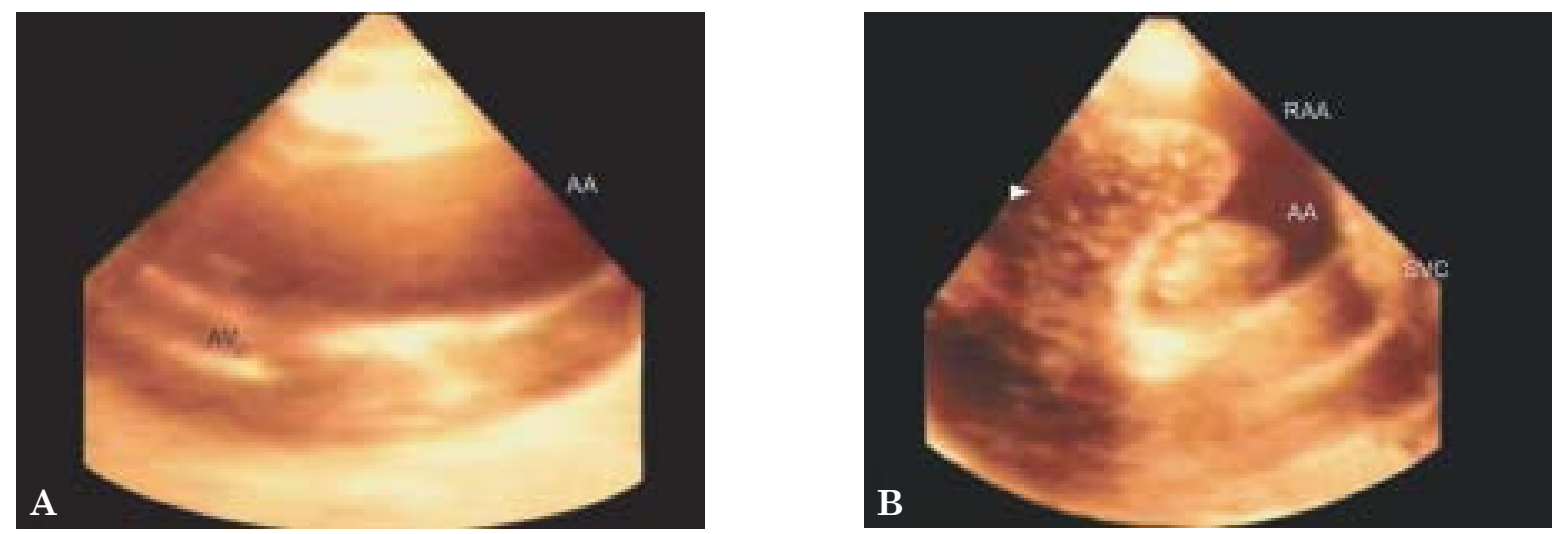

Figs.-12A \& B: Three-dimensional right parasternal transthoracic echocardiographic examination of ascending aorta and right atrial appendage (RAA). (A) Enlarged $A A ;(B) R A A$ and its relationship to the superior vena cava (SVC) are demonstrated. The arrowhead points to contrast signals in RAA following an intravenous bubble study. A small portion of AA is imaged between RAA and SVC. Source: Reproduced with permission from reference 3.
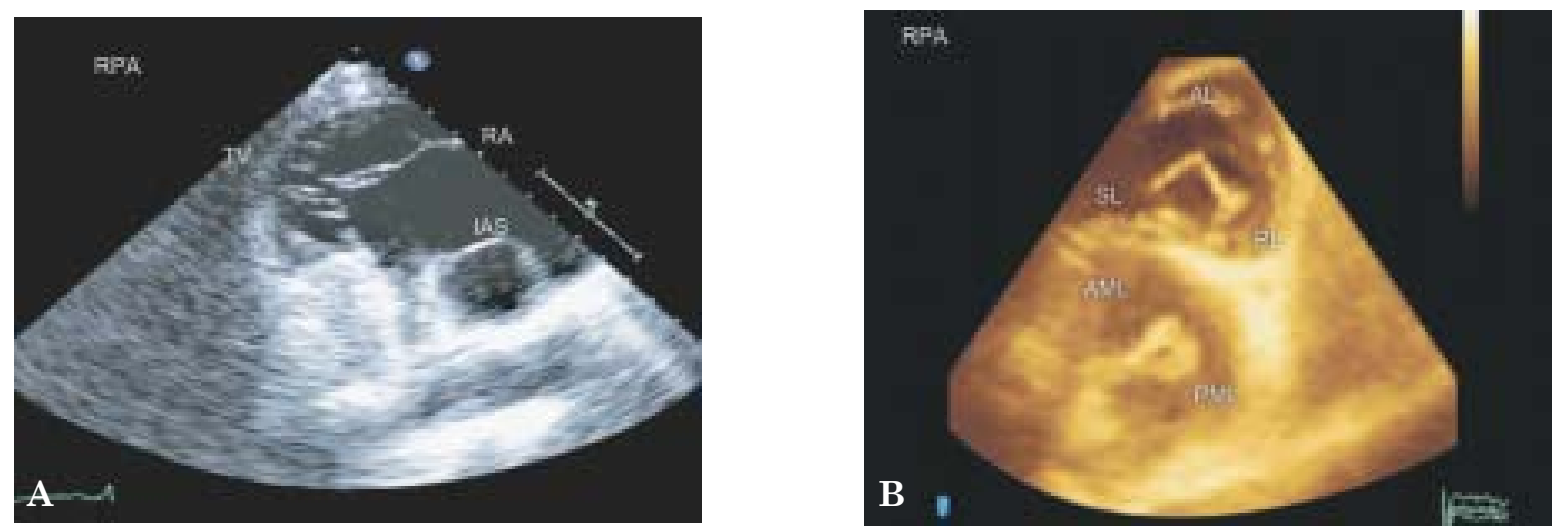

Figs.-13 A \&B: Right parasternal approach (RPA). (A) Two dimensional transthoracic echocardiography. The coronary sinus is seen entering the right atrium (RA). The Thebesian valve is not detected. (B) The short-axis view demonstrates all three leaflets of the tricuspid valve (TV) and both leaflets of the mitral valve. (AL: Anterior leaflet; AML: Anterior mitral leaflet; IAS: Interatrial septum; PL: Posterior leaflet; PML: Posterior mitral leaflet; SL: Septal leaflet).
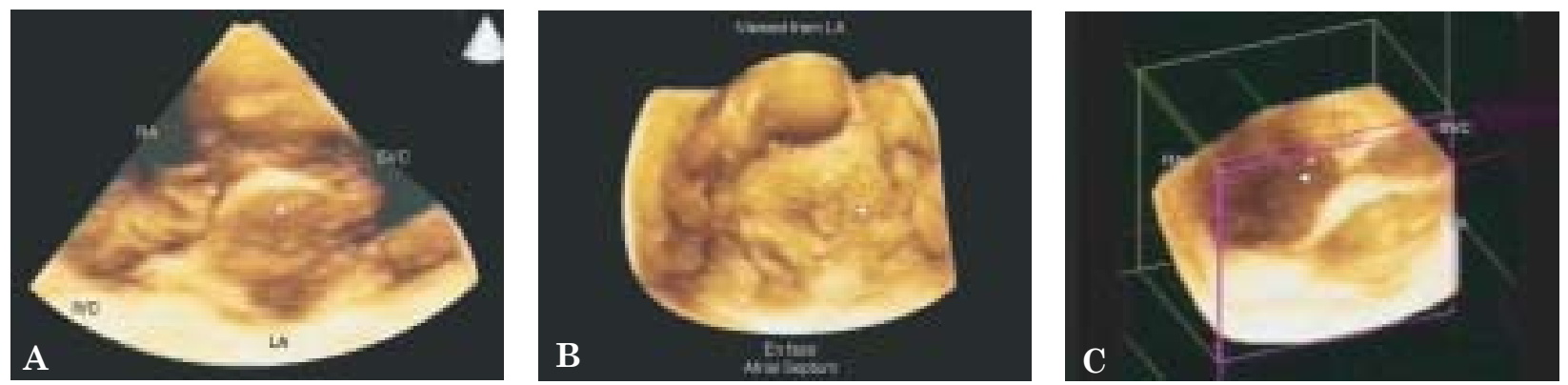

Figs.-14A to C: Three-dimensional transthoracic echocardiographic visualization of the valve of foramen ovale. Right parasternal approach. (A) The arrowhead points to the atrial septum; (B) En face view of the atrial septum from the left atrial (LA) side shows the valve of foramen of ovale (arrowhead) in the open positions. In the closed position, it completely covers the foramen ovale; $(C)$ The arrowhead points to a mobile flap of tissue at the junction of the superior vena cava (SVC) and the right atrium (RA) representing a remnant of right-sided sinus venosus valve. Source: Reproduced with permission from reference 5 . 


\section{Right and Left Supraclavicular Examination}

This is performed by placing the transducer above the right and left clavicles. The superior vena cava further upstream from its junction with the right atrium is easily visualized using the right supraclavicular examination approach. Both the left and right innominate veins that join to form the superior vena cava may also be delineated as well as a venous valve often present at the junction with the superior vena cava. Standard textbooks of anatomy do not mention this venous valve, but it is frequently noted echocardiographically and has sometimes been mistaken for a dissection flap in the aorta or an aortic branch. The left supraclavicular window is also useful to examine the left innominate vein and the adjoining arterial vessels. This view is also useful to detect a leftsided superior vena cava. Catheters, pacemaker wires, thrombi, and other mass lesions present in these veins are readily visualized and evaluated. Figures 15 to 18 demonstrate normal and abnormal findings imaged from the right and left supraclavicular approaches using two-dimensional and live/real time, three-dimensional echocardiography. ${ }^{1-3}$

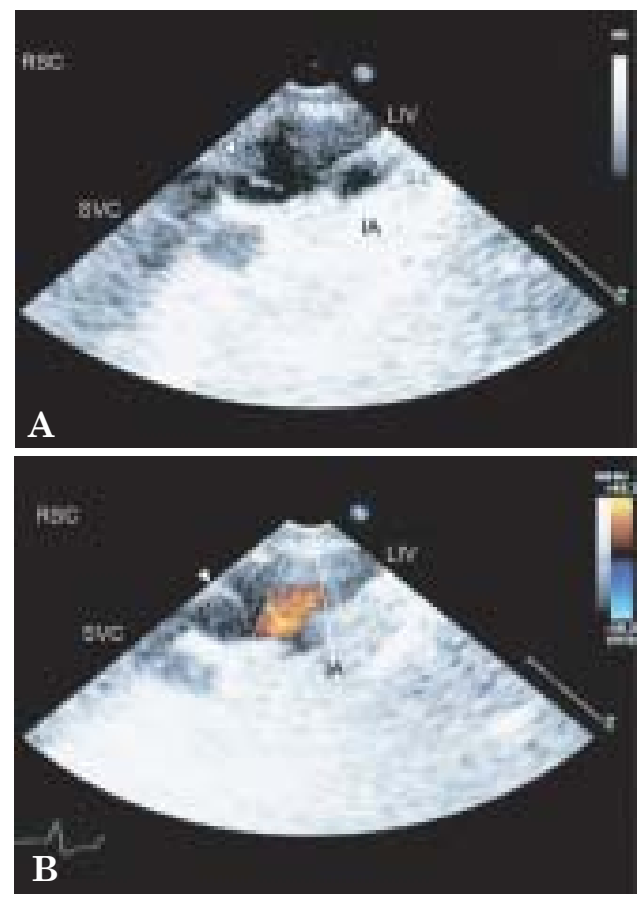

Figs.-15A \& B: Two-dimensional transthoracic echo-cardiography. Right supraclavicular approach (RSC). (A) shows the left innominate vein (LIV) draining into the superior vena cava $(S V C)$. The arrowhead points to a mildly thickened venous valve at the junction of LIV and SVC; (B) Color Doppler examination shows flow signals (red) in the innominate artery (IA) and a venous valve (arrowhead).

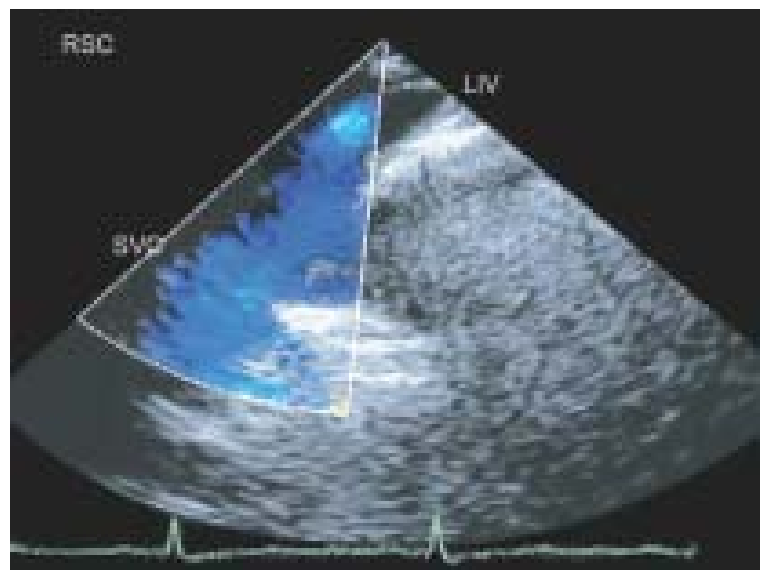

Fig.-16: Two-dimensional transthoracic echocardio-graphy. Right supraclavicular approach (RSC). Color Doppler examination shows flow signals (blue) in the SVC.
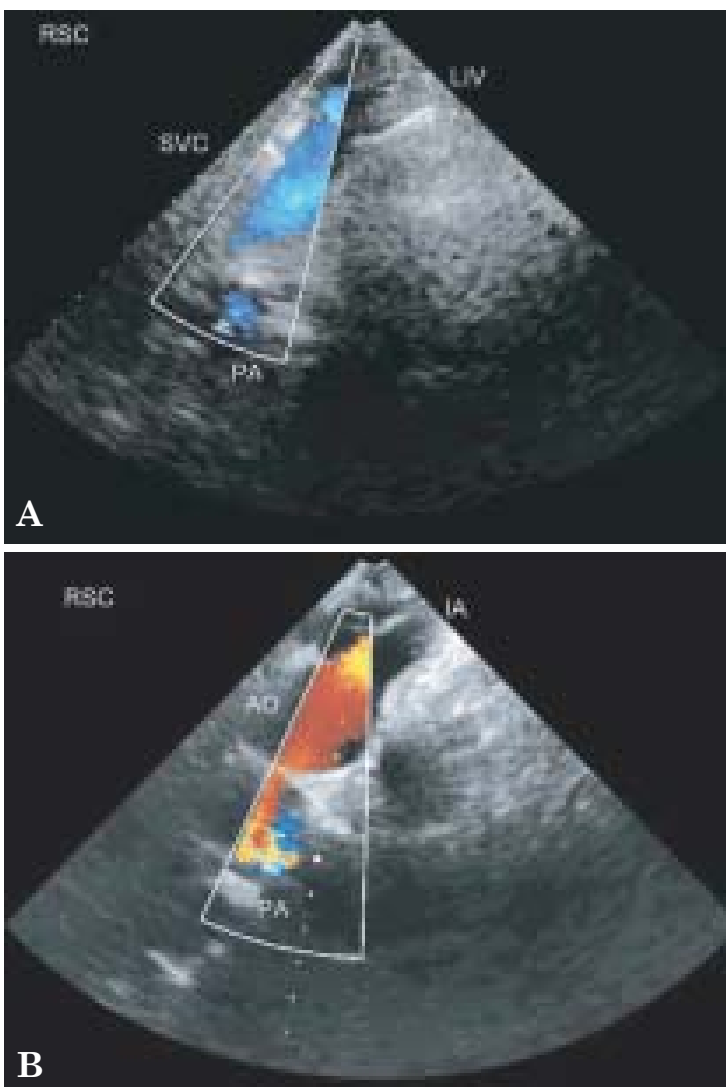

Figs.-17A and B: Two-dimensional transthoracic echocardiography. The right supraclavicular (RSC) approach. (A) Color Doppler examination shows flow signals (blue) in the superior vena cava (SVC) and the pulmonary artery (PA); (B) The transducer was angled to image the ascending aorta $(A O)$ and the innominate artery (IA) from this approach. (LIV: Left innominate vein). 

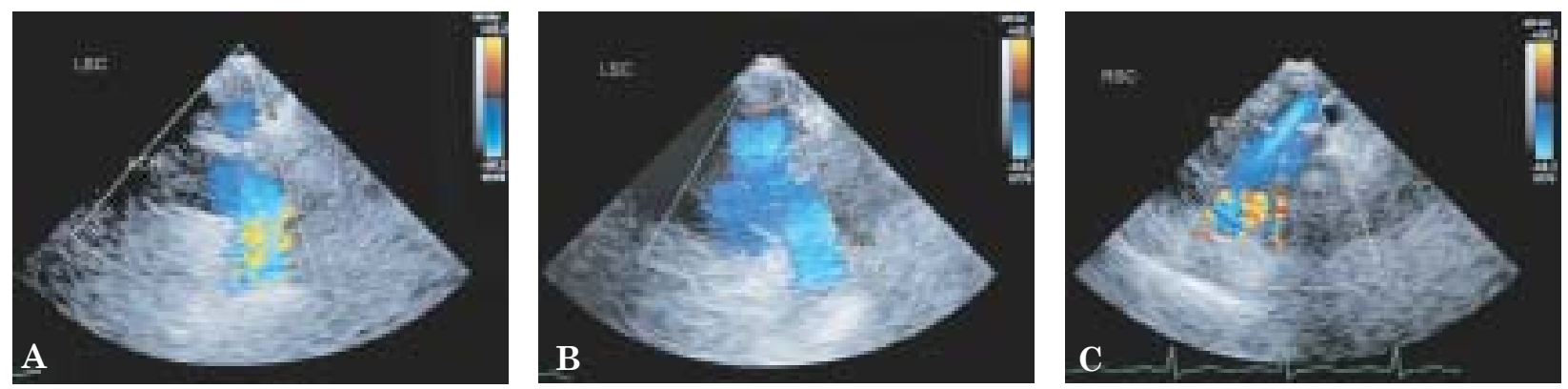

Figs.-18A to C: Left-sided superior vena cava. Two-dimensional transthoracic echocardiography. (A\&B) Left supraclavicular (LSC) examination shows the left-sided superior vena cava (SVC) (L) superior to the aortic arch and the descending thoracic aorta. (C) Right supraclavicular (RSC) examination. The right $S V C$ is also present in this patient. (ACH: Aortic arch; AO: Aorta; DA: Descending thoracic aorta; LA: Left atrium; LV: Left ventricle; PA: Pulmonary artery; RV: Right ventricle).

\section{Left Parasternal and Apical Planes for Examination of Coronary Arteries}

Even though the orifices and proximal portions of both left and right coronary arteries can be evaluated using the aortic short-axis view, examination of other segments of the coronary arteries require the use of nonstandard planes. Keeping the transducer position in-between those used for standard parasternal and apical views and angling it inferiorly such that the left ventricular cavity practically disappears may bring into view a long segment of the mid-left anterior descending coronary artery. This can be evaluated for the presence of atherosclerotic plaques and lumen stenosis in B-mode as well as using color Doppler and pulsed Doppler interrogation. Coronary artery flow reserve can be estimated by giving intravenously dipyridamole or adenosine during pulsed Doppler interrogation. An increase in peak velocity of at least four times the baseline value indicates good flow reserve and absence of significant stenosis in the relevant coronary territory whereas a lower value portends insufficient flow reserve and significant obstructive disease. Apical and para-apical views have been used to identify and assess flow reserve in visualized segments of circumflex and posterior descending coronary arteries.
Occasionally, intramyocardial coronary vessels have also been detected using the parasternal approach especially in the presence of external compression, which results in a significant increase in their flow velocity. Figures 19 and 23 depict normal and abnormal findings acquired using two-dimensional and live/real time, threedimensional echo-cardiography. ${ }^{6-8}$

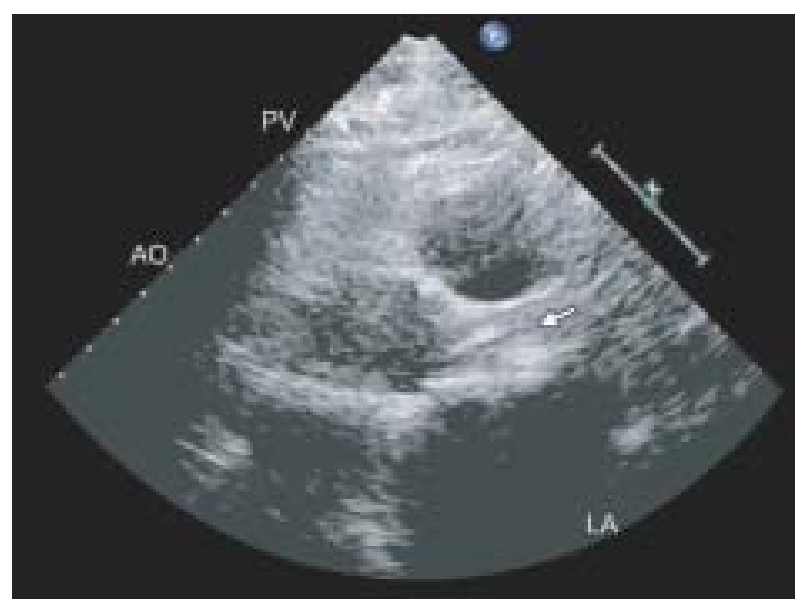

Fig.-19: Left main coronary artery. Twodimensional transthoracic echocardiography. Parasternal short-axis view at the level of the aorta (AO) shows a small, left main coronary artery (arrow) with luminal irregularity due to nonobstructive atherosclerotic plaques. 

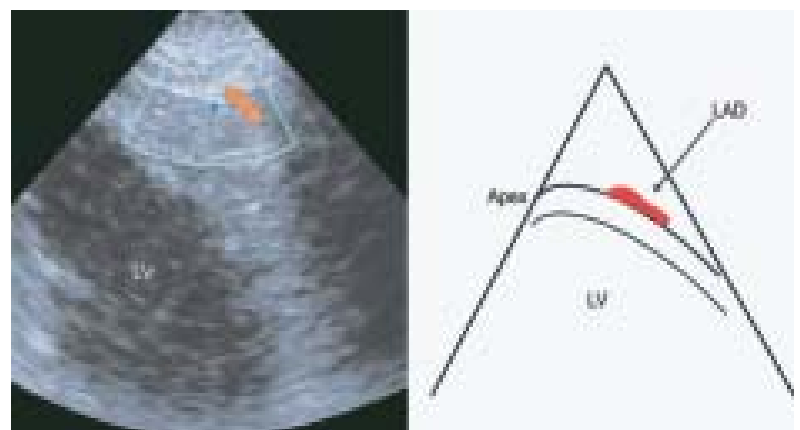

(A)
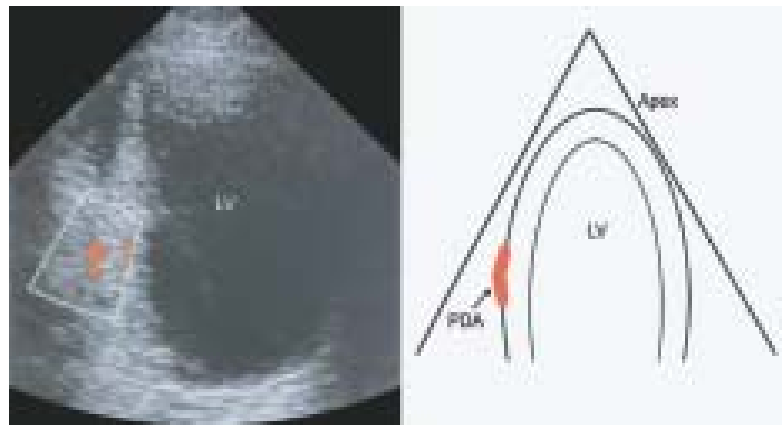

(B)
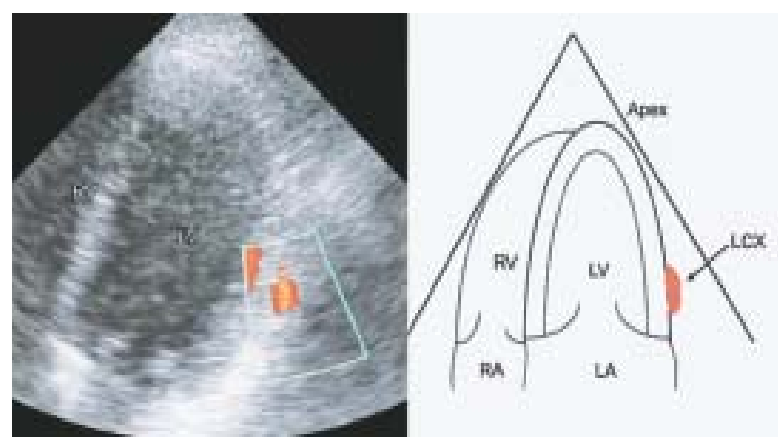

(C)

Figs.-20A to C: (A) Transthoracic color Doppler echocardiography demonstrating coronary blood flow (left) and schematic representation (right) in the distal LAD; (B) Transthoracic color Doppler echocardiography demonstrating coronary blood flow (left) and schematic representation (right) in the PDA; (C) Transthoracic color Doppler echocardiography demonstrating coronary blood flow (left) and schematic representation (right) in the LCX. (LAD: Left anterior descending artery; LCX: Left circumflex artery; PDA: Posterior descending artery). Source: Reproduced with permission from reference 7 .
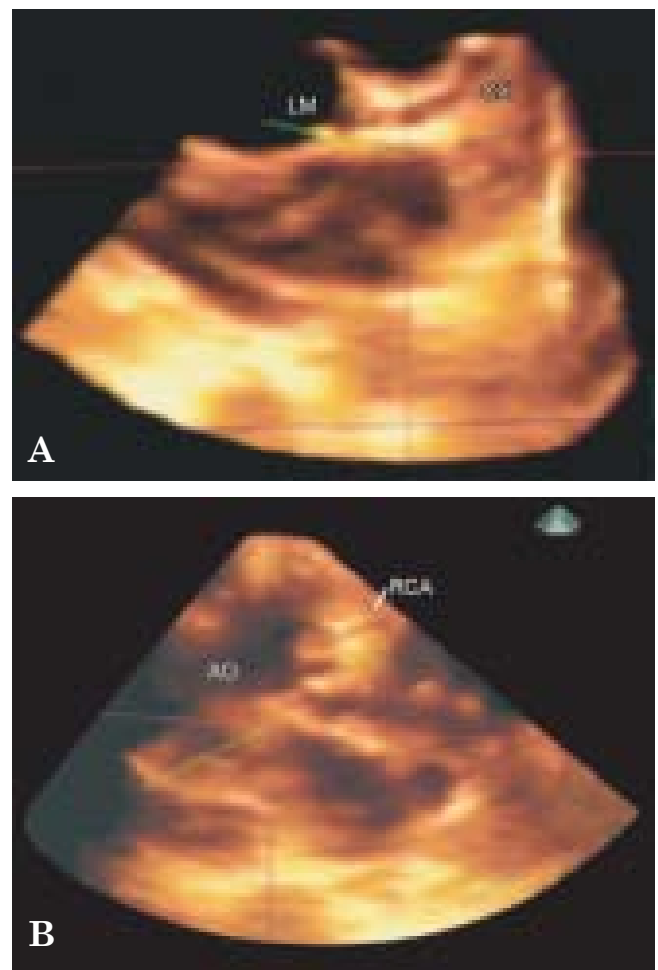

Figs.-21A and B: Transthoracic echocardiography of coronary arteries of a 69-year-old female. (A) The pyramidal section has been cropped to show normal left main (LM) and proximal circumflex $(C X)$ coronary arteries. The left anterior descending coronary artery is located in a different plane; (B) Another section in the same patient demonstrates a normal proximal right coronary artery (RCA). (AO: Aorta). Source: Reproduced with permission from reference 8 .

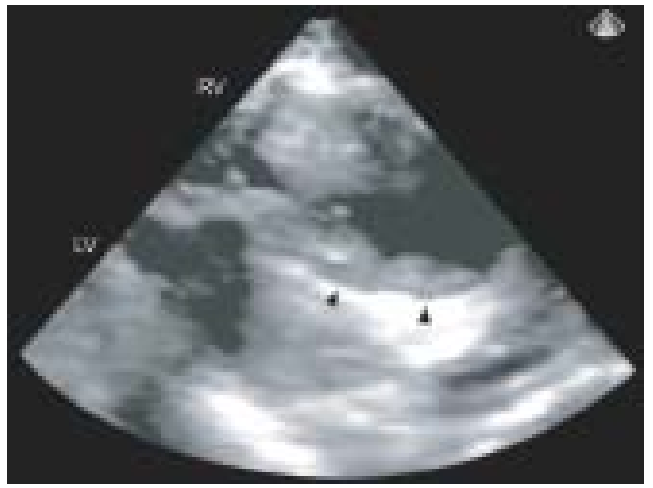

Fig.-22: Three-dimensional transthoracic echocardio-graphy of coronary arteries of a 26year-old male. The arrowheads demonstrate the midportion of the left anterior descending coronary artery (LAD) located in the anterior interventricular groove. (LV: Left ventricle; $R V$ : Right ventricle). Source: Reproduced with permission from reference 8. 

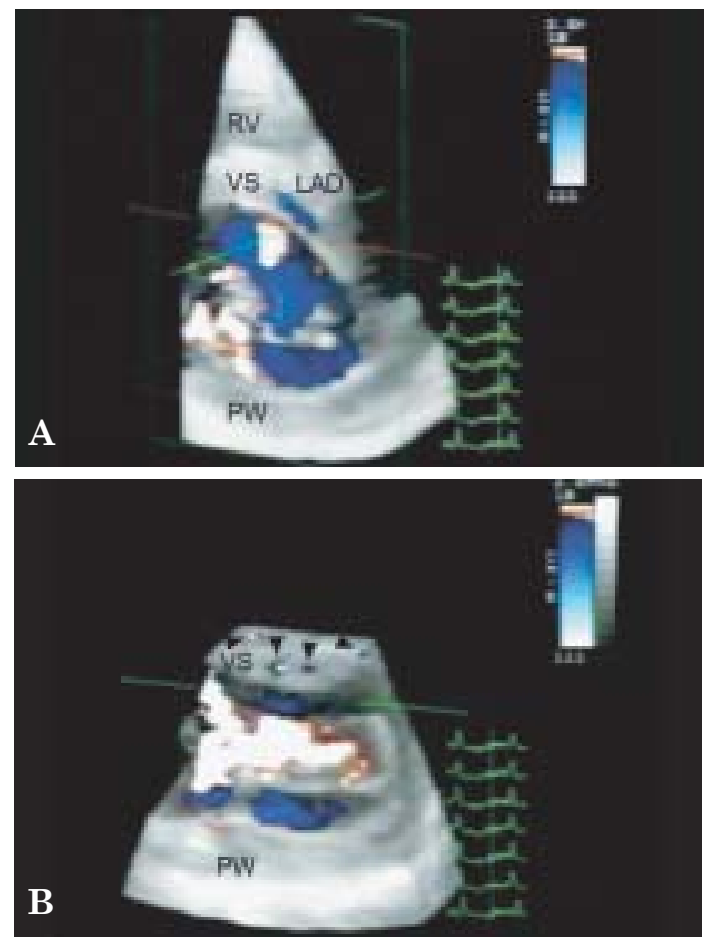

Figs.-23 A \& B: Three-dimensional transthoracic echocardiography of coronary arteries of a 30-year-old male, status postorthotopic cardiac transplantation. (A) Three-dimensional color Doppler flow imaging demonstrates a segment of $L A D$ adjacent to the ventricular septum (VS). This segment was not visualized by B-mode, three dimensional imaging; $(B)$ The arrowhead points to multiple septal perforators imaged in the short-axis within the VS. (AO: Aorta; D1: First diagonal branch of LAD; D2: Second diagonal branch of LAD; LM: Left main; $R V$ : Right ventricle; RVOT: Right ventricular outflow tract). Source: Reproduced with permission from reference 8 .

\section{Examination of Left Atrial Appendage}

Although most books do not describe the technique to examine the left atrial appendage, this structure can be easily detected in most of the patients using conventional planes. Aortic short-axis and mitral or left ventricular-pulmonic planes can be utilized to find the appendage with slight leftward and superior angulation. Minimal adjustment of the transducer is needed to examine it fully from the base to the tip. Apical views are also useful to find the appendage. One generally begins with the four chamber view and from this position, rotation of the probe toward the five-chamber view or two-chamber view will bring the appendage into view. The appendage is enlarged and, therefore, more easily visualized in patients with atrial fibrillation and mitral stenosis, two conditions in which it is important to rule out a thrombus. In patients with good acoustic windows and absolute or relative contraindications for transesophageal echocardiography, two-dimensional echocardiography has been successfully used to assess the appendage for the presence of a clot. In these cases, live/real time, three-dimensional echocardio-graphy can increase the confidence level in ruling out a clot. This is because the whole of the left atrial appendage can be acquired in the three-dimensional dataset, which can then be cropped sequentially in a systematic manner. Thus, short-axis and long-axis sections of the appendage can be viewed to diagnose or rule out the presence of a clot. Individual lobes of the appendage may also be examined using this technique. We have also had patients with atrial fibrillation in whom a clot was diagnosed by transesophageal echocardio-graphy and the patient placed on anticoagulant therapy for several weeks without any change in its size. Subsequently, combined two- and threedimensional echocardiography showed the alleged clot to be a prominent pectinate muscle viewed in the short axis. No clot was found, and the patient underwent successful ablation. Figures $24 \& 25$ demonstrate normal and abnormal findings acquired using twodimensional and live/real time, threedimensional echocardiography. ${ }^{1,9}$

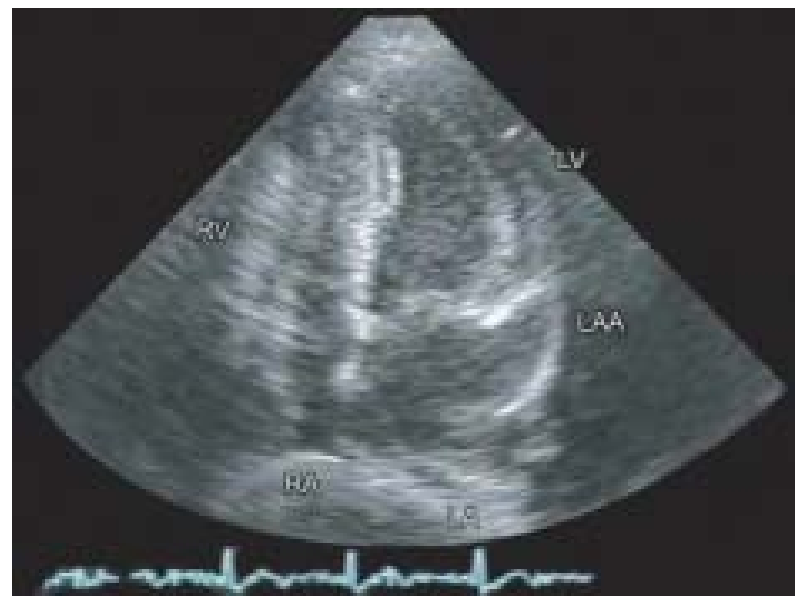

Fig.-24: Left atrial appendage (LAA). Twodimensional transthoracic echocardiography. $L A A$ is visualized using the apical fourchamber view and rotating the probe toward the fivechamber view. $L A$, left atrium; $L V$, left ventricle; $R A$, right atrium; $R V$, right ventricle. 

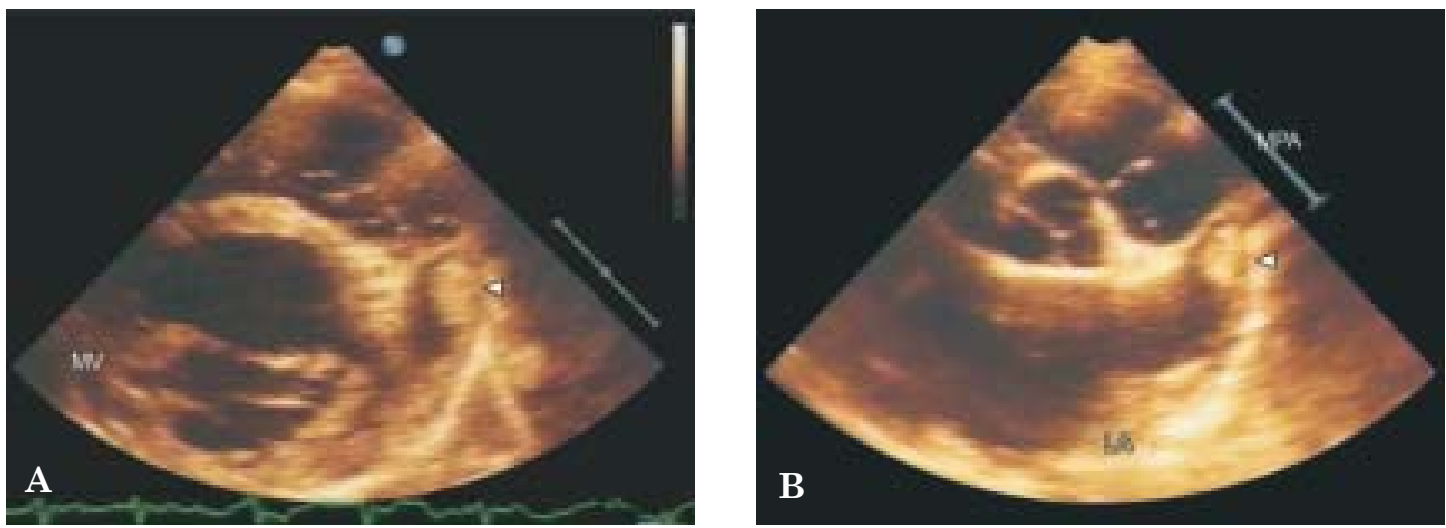

Figs.-25 A \& B Left ventricular (A) and aortic (B) short-axis views. The arrowheads show a clot in the left atrial appendage. Cropping of the clot shows no echo lucency within it consistent with the absence of lysis. This is most likely because the clot is fresh and the natural anticoagulant mechanisms present in the blood; have not yet had enough time to act on it. (LA: Left atrium; MPA: Main pulmonary artery; MV: Mitral valve). Source: Reproduced with permission from reference 9.

\section{Examination from the back}

The presence of a large left or right pleural effusion opens up an acoustic window from the back, and the heart can be examined by placing the transducer in the posterior intercostal spaces. This is best accomplished by examining the patient in the sitting position. Four-chamber views of the heart are easily obtained when the effusion is large. A distinct advantage of this approach is assessment of a large segment of the descending thoracic aorta, which is difficult to visualize using standard transthoracic windows. This approach has also been used in patients with pleural effusion undergoing percutaneous interventions in the cardiac catheterization laboratory since, unlike the anterior transthoracic approach; it does not cause any significant interference with the procedure. The main utility of this window is in making a confident diagnosis of right- or leftsided pleural effusion and distinguishing it from other large fluid collections such as ascites and coexisting pericardial effusion. Figure 26 demonstrate normal and abnormal findings acquired using two-dimensional and live/real time, three-dimensional echocardiography.
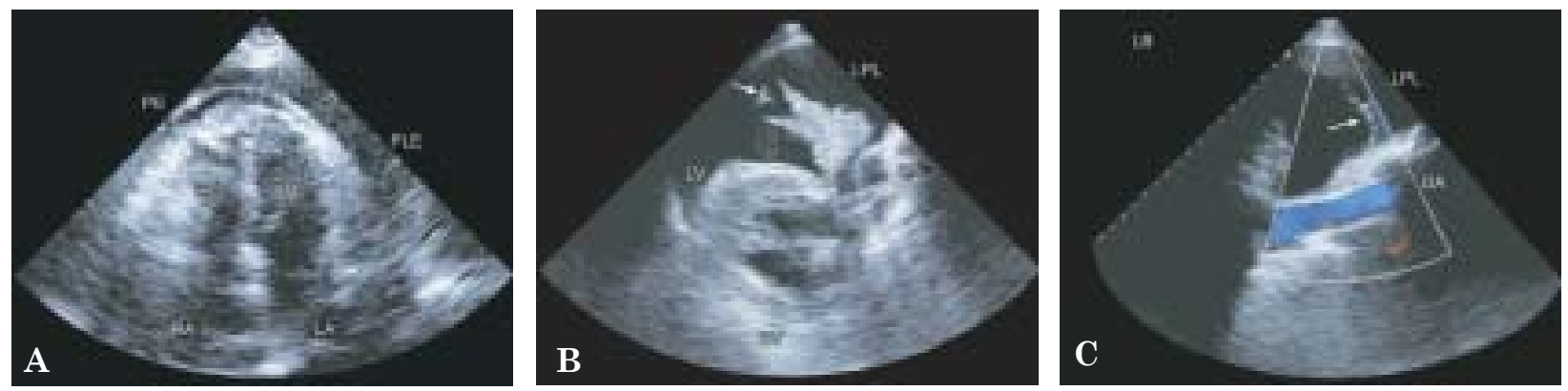

Figs.-26 A to C: Two-dimensional transthoracic echocardiographic examination from the back in a 77-year-old patient with lymphoma and bilateral pleural effusion. (A) Apical four-chamber view showing left pleural effusion (LPL) and pericardial effusion (PE); (B) Examination of heart from $L B$. The arrow shows a part of the collapsed lung. Both the left $(L V)$ and the right (RV) ventricles as well as the descending thoracic aorta (DA) are visualized through the pleural effusion; (C) Color Doppler examination showing flow signals (blue) in the DA imaged from LB. The arrow points to a portion of the collapsed lung. (L: Liver; LA: Left atrium; RA: Right atrium; S: Spleen). 

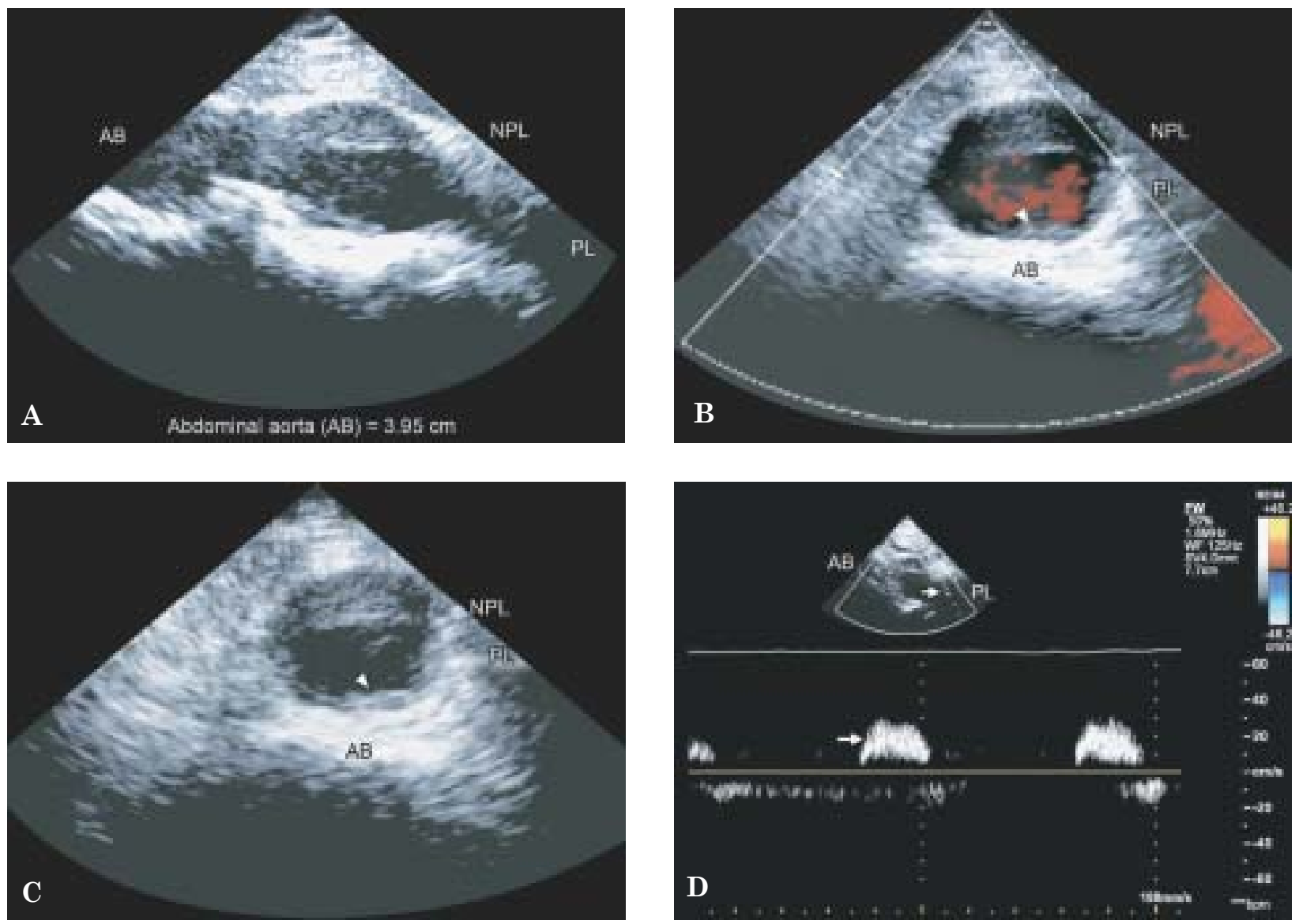

Figs.-27 A to D: Two-dimensional subcostal echocardiography. (A) shows a long-axis view of the abdominal aorta (AB). It is enlarged measuring $3.95 \mathrm{~cm}$ and demonstrates a dissection flap with perfusing (PL) and nonperfusing lumens (NPL); (B and C) Short-axis viewof the abdominal aorta using B-mode and color Doppler examination, respectively. The arrowhead points to an atherosclerotic plaque within the aorta. The NPL is clotted; (D) The upper arrow points to a Doppler cursor line passing through $A B$, and the lower arrow showspulsatile flow signals obtained from the $A B$ using pulsed wave Doppler.

\section{Abdominal Examination}

Subcostal examination is a part of standard echocardiographic examination protocol. However, often scant attention is paid to examining the abdominal aorta. This is an important vessel that can be involved in aneurysm formation or dissection that can go undetected clinically because often the patient may not present with any symptoms. Therefore, it is useful to routinely attempt to image the abdominal aorta in all patients undergoing echocardiography. Other abnormal findings such as gall stones, which can be a causative factor in patients presenting with chest pain, should also be reported. Figure 27 depict normal and abnormal findings acquired using twodimensional and live/real time, threedimensional echocardiography. ${ }^{1,10}$

\section{References}

1. Cooper JW, Aggarwal KK, Fan P, et al. Echocardiographic examination technique: a new practical approach. In: Nanda NC, editor. Textbook of Color Doppler Echocardiography. 2nd ed. Philadelphia, PA: Lea \& Febiger; 1989: 99-115.

2. Nanda NC, Hsiung MC, Miller AP, et al. editors. How to do a 3D echocardiogram: examination protocol and normal anatomy. In: Live/Real Time 3D Echocardiography. Oxford, England: Blackwell; 2010: 23-54.

3. Patel V, Nanda NC, Upendram S, et al. Live threedimensional right parasternal and supraclavicular transthoracic echocardiographic examination. Echocardiography 2005; 22(4):349-360.

4. Upendram S, Nanda NC, Vengala S, et al. Live threedimensional transthoracic echocardiographic assessment of thrombus in the innominate veins and superior vena cava utilizing right parasternal and 
supraclavicular approaches. Echocardiography 2005; 22(5): 445-449.

5. Panwar SR, Perrien JL, Nanda NC, et al. Real time/ threedimensional transthoracic echocardiographic visualization of the valve of foramen ovale. Echocardiography 2007; 24(10):1105-1107.

6. Nanda NC, Hsiung MC, Miller AP, et al. (Eds). Ischemic heart disease. In: Live/Real Time 3D Echocardiography. Oxford, England: Blackwell; 2010: 128-36.

7. Murata E, Hozumi T, Matsumura Y, et al. Coronary flow velocity reserve measurement in three major coronary arteries using transthoracic Doppler echocardiography. Echocardiography 2006;23(4):279-286.
8. Vengala S, Nanda NC, Agrawal G, et al. Live threedimensional transthoracic echocardiographic assessment of coronary arteries. Echocardiography 2003; 20(8): 751-754.

9. Karakus G, Kodali V, Inamdar V, et al. Comparative assessment of left atrial appendage by transesophageal and combined two- and three-dimensional transthoracic echocardiography. Echocardiography 2008;25(8):918-924.

10. Daly DD Jr, El-Shurafa H, Nanda NC, et al. Does the routine echocardiographic exam have a role in the detection and evaluation of cholelithiasis and gallbladder wall thickening? Echocardiography 2012; 29(8): 991-996. 